\title{
A BIRATIONAL ANABELIAN RECONSTRUCTION THEOREM FOR CURVES OVER ALGEBRAICALLY CLOSED FIELDS IN ARBITRARY CHARACTERISTIC
}

\author{
MARTIN LÜDTKE
}

\begin{abstract}
The aim of Bogomolov's programme is to prove birational anabelian conjectures for function fields $K \mid k$ of varieties of dimension $\geq 2$ over algebraically closed fields. The present article is concerned with the 1-dimensional case. While it is impossible to recover $K \mid k$ from its absolute Galois group alone, we prove that it can be recovered from the pair (Aut $(\bar{K} \mid k)$, Aut $(\bar{K} \mid K)$ ), consisting of the absolute Galois group of $K$ and the larger group of field automorphisms fixing only the base field.
\end{abstract}

\section{Contents}

1. Introduction 1

2. Injectivity 2

3. A Galois-Type Correspondence for Transcendental Field Extensions 3

4. Detecting the Rationality of Function Fields 7

5. Detecting Decomposition Groups 8

6. Proof of Main Theorem 12

References $\quad 15$

\section{INTRODUCTION}

The aim of the birational anabelian program initiated by Bogomolov [Bog91] at the beginning of the 1990's is to recover function fields $K \mid k$ of dimension $>1$ over algebraically closed fields from their absolute Galois group $G_{K}$. This cannot be possible in the one-dimensional case since then $G_{K}$ is profinite free of rank $|k|$ by results of Harbater [Har95] and Pop [Pop95], containing therefore almost no information about $K$. We show however that $K \mid k$ can be recovered if in addition to $G_{K}$ also the larger automorphism group $\operatorname{Aut}(\bar{K} \mid k) \supseteq G_{K}$ fixing only the base field is provided. On the way, we prove a Galois-type correspondence for transcendental field extensions and give a group-theoretic characterisation of stabiliser subgroups for $\operatorname{PGL}(2, k)$ acting on $\mathbb{P}^{1}$.

We use the following notation: Let $k$ be an algebraically closed base field. A function field $K \mid k$ is a finitely generated field extension. Its dimension is defined as the transcendence degree $\operatorname{trdeg}(K \mid k)$. If $F:=\bar{K}$ is an algebraic closure, we write $G_{F \mid k}:=\operatorname{Aut}(F \mid k)$ and denote the absolute Galois group of $K$ by $U_{K}:=\operatorname{Aut}(F \mid K) \cong \operatorname{Gal}\left(K^{\operatorname{sep}} \mid K\right)$. The group $G_{F \mid k} \subseteq \operatorname{Map}(F, F)$ is endowed with the compact-open topology for discrete $F$, making $U_{K}$ an open subgroup whose induced topology agrees with the usual profinite Krull topology.

Theorem A. Let $k$ be an algebraically closed field and $K \mid k$ a 1-dimensional function field with algebraic closure $F=\bar{K}$. If $\left(k^{\prime}, K^{\prime}, F^{\prime}\right)$ is another such triple, then the natural map

$$
\Phi: \operatorname{Isom}\left(F^{\prime}\left|K^{\prime}\right| k^{\prime}, F|K| k\right) \longrightarrow \operatorname{Isom}\left(\left(G_{F \mid k}, U_{K}\right),\left(G_{F^{\prime} \mid k^{\prime}}, U_{K^{\prime}}\right)\right)
$$

is a bijection.

The right hand side consists of isomorphisms of topological groups $G_{F \mid k} \stackrel{\sim}{\longrightarrow} G_{F^{\prime} \mid k^{\prime}}$ which restrict to an isomorphism between the open subgroups $U_{K} \cong U_{K^{\prime}}$. An isomorphism between 
field towers $F^{\prime}\left|K^{\prime}\right| k^{\prime}$ and $F|K| k$ is by definition an isomorphism $\sigma: F^{\prime} \stackrel{\sim}{\longrightarrow} F$ restricting to isomorphisms $K^{\prime} \cong K$ and $k^{\prime} \cong k$. The natural map in the theorem assigns to such $\sigma$ the isomorphism $\Phi(\sigma):\left(G_{F \mid k}, U_{K}\right) \stackrel{\sim}{\longrightarrow}\left(G_{F^{\prime} \mid k^{\prime}}, U_{K^{\prime}}\right)$ given by

$$
\Phi(\sigma)(\tau)=\sigma^{-1} \circ \tau \circ \sigma .
$$

It is useful to not single out one function field in $F \mid k$ but rather work with the totality of them and study their interplay that comes from inclusions between them, or equivalently from morphisms between their complete nonsingular models. We therefore prove the following variant which, as proved at the end of Section 3, implies Theorem A.

Theorem B. Let $F \mid k$ be an extension of algebraically closed fields of transcendence degree 1. If $F^{\prime} \mid k^{\prime}$ is another extension of algebraically closed fields, the natural map

$$
\Phi: \operatorname{Isom}^{i}\left(F^{\prime}\left|k^{\prime}, F\right| k\right) \longrightarrow \operatorname{Isom}\left(G_{F \mid k}, G_{F^{\prime} \mid k^{\prime}}\right)
$$

is a bijection.

Here, Isom ${ }^{i}$ denotes the set of isomorphisms up to Frobenius twists, i.e. identifying $\sigma \sim$ Frob $^{n} \circ \sigma$ for $n \in \mathbb{Z}$ if $\operatorname{char}(k)=\operatorname{char}\left(k^{\prime}\right)=p>0$, where Frob $\in \operatorname{Isom}(F|k, F| k)$ is $\operatorname{Frob}(x)=x^{p}$.

The present article is heavily based on [Rov03]. It contains however several new aspects, most notably extending the results to function fields of positive characteristic. This required a Galois-type correspondence theorem for certain transcendental field extensions (Theorem 3.3). Moreover, we include the details of how the stabiliser subgroups of $\operatorname{PGL}(2, k)$ acting on $\mathbb{P}^{1}$ are group-theoretically distinguished (Lemma 5.6) and present a simplified way of detecting decomposition groups (Lemma 5.7). We also take an alternative route to reconstruct the function fields from there, namely via the reconstruction of ramification indices and principal divisors, whereas the approach of [Rov03] is based on linear systems.

Acknowledgements. The author would like to thank Jakob Stix for careful proofreading and many helpful comments, Armin Holschbach for numerous discussions and Alexander Schmidt for supervising the master's thesis from which this article originated.

\section{INJECTIVITY}

We start by quickly treating the easier part of Theorem A, namely the question of injectivity. In fact, we show the following stronger statement.

Theorem 2.1. Let $K$ be a function field of dimension $\geq 1$ over an algebraically closed field $k$, let $F$ be its algebraic closure and $U_{K}=\operatorname{Aut}(F \mid K)$ its absolute Galois group. If $\left(k^{\prime}, K^{\prime}, F^{\prime}\right)$ is another such triple, then the natural map

$$
\operatorname{Isom}\left(F^{\prime}\left|K^{\prime}, F\right| K\right) \longrightarrow \operatorname{Isom}\left(U_{K}, U_{K^{\prime}}\right)
$$

is injective.

Note that the function fields $K$ and $K^{\prime}$ are allowed to have any dimension $\geq 1$, and on the right hand side we have isomorphisms between $U_{K}$ and $U_{K^{\prime}}$ rather than isomorphisms of pairs $\left(G_{F \mid k}, U_{K}\right),\left(G_{F^{\prime} \mid k^{\prime}}, U_{K^{\prime}}\right)$. Thus the statement applies also to situations that Bogomolov's conjecture is concerned with. Our proof uses a valuation theoretic result of F. K. Schmidt and is similar to that of the last lemma in [Pop90].

Recall that a rank 1 valuation on a field $K$ is a map $v: K \rightarrow \mathbb{R} \cup\{\infty\}$ satisfying
(a) $v(x)=\infty \Leftrightarrow x=0$
(b) $v(x y)=v(x)+v(y)$;
(c) $v(x+y) \geq \min \{v(x), v(y)\}$; 
and moreover $v \not \equiv 0$ on $K^{\times}$. It is a discrete valuation if its value group $v\left(K^{\times}\right)$is a discrete subgroup of $\mathbb{R}$. Two rank 1 valuations $v, w$ on $K$ are equivalent, written $v \sim w$, if $w=\lambda v$ for some $\lambda>0$. The automorphism group $\operatorname{Aut}(K)$ acts on the set of rank 1 valuations on $K$ by the rule $\tau v=v \circ \tau^{-1}$. The decomposition group of $v$ over a subfield $k \subseteq K$ is defined as

$$
Z_{v}(K \mid k)=\{\tau \in \operatorname{Aut}(K \mid k) \mid \tau v \sim v\} .
$$

The field $K$ is henselian with respect to the valuation $v$ if $v$ extends uniquely to every algebraic extension of $K$.

Lemma 2.2. Let $K$ be a field, $F=\bar{K}$ its algebraic closure and $v, w$ two rank 1 valuations on $F$ with decomposition groups $Z_{v}, Z_{w}$ over $K$. If $v$ and $w$ are inequivalent, then

$$
Z_{v} \cap Z_{w}=1 .
$$

Proof. The fixed field $F^{Z_{v} \cap Z_{w}}$ is henselian with respect to the restrictions of $v$ and $w$, hence is separably closed by a theorem of F. K. Schmidt (cf. [EP05], Thm. 4.4.1). Thus, $F^{Z_{v} \cap Z_{w}}=F$ and $Z_{v} \cap Z_{w}=1$.

Lemma 2.3. Let $K$ be a field and $\sigma \in \operatorname{Aut}(K)$. If there exists a proper subfield $k \subset K$ such that $\sigma x / x \in k^{\times}$for all $x \in K^{\times}$, then $\sigma=\mathrm{id}$.

Proof. Put $a(x)=\sigma x / x \in k^{\times}$. By additivity of $\sigma$, we have

$$
a(x) x+a(y) y=a(x+y) x+a(x+y) y
$$

for all $x, y \in K^{\times}$with $x+y \neq 0$. For $k$-linearly independent $x$ and $y$, we obtain $a(x)=a(x+y)=$ $a(y)$. For linearly dependent $x$ and $y$ we find $a(x)=a(y)$ as well, by comparing with an element of $K^{\times}$which is linearly independent from $x$ and $y$. So we have $\sigma x=a x$ for some $a \in k^{\times}$which does not depend on $x$. Since $\sigma(1)=1$, we have $a=1$.

Proof of Theorem 2.1. It suffices to show that if $\sigma \in \operatorname{Aut}(F)$ is an automorphism with $\sigma K=K$ for which $\sigma^{-1}(-) \sigma=$ id in $\operatorname{Aut}\left(U_{K}\right)$, then $\sigma=$ id. Consider a rank 1 valuation $v$ of $F$ which is discrete on $K$. Its decomposition group $Z_{v}$ over $K$ is nontrivial since $v$ ramifies in $K^{\text {sep }} \mid K$, e.g. by adjoining $\ell$-th roots of a uniformiser for $\ell \neq \operatorname{char}(k)$. The decomposition group of $\sigma^{-1} v$ is $Z_{\sigma^{-1} v}=\sigma^{-1} Z_{v} \sigma=Z_{v}$, hence $\sigma^{-1} v=\lambda v$ for some $\lambda>0$ by Lemma 2.2. Since $\sigma K=K$, the valuations $v$ and $\lambda v$ have the same value group on $K$. As this value group is discrete, we must have $\lambda=1$, thus $\sigma^{-1} v=v$. So we have $v(\sigma x / x)=0$ for all $x \in F^{\times}$and all rank 1 valuations $v$ on $F$ which are discrete on $K$. We claim that this implies $\sigma x / x \in k^{\times}$, so that we are done by Lemma 2.3. Indeed, otherwise we find a transcendence basis $T=\left(t_{1}, \ldots, t_{n}\right)$ of $F \mid k$ with $t_{1}=\sigma x / x$ and a rank 1 valuation $v$ on $F$ extending the $t_{1}$-adic valuation on $k(T)$. Then $v$ is discrete on $k(T)$ and since the composite field $K(T)$ is a common finite extension of $K$ and $k(T)$, it is also discrete on $K$. But by construction $v(\sigma x / x) \neq 0$, contradiction!

\section{A Galois-Type Correspondence for Transcendental Field Extensions}

Let $k$ be a field and $F \mid k$ a field extension with $F$ algebraically closed. We prove a generalised Galois correspondence for such extensions and apply it in the case where $k$, too, is algebraically closed and $\operatorname{trdeg}(F \mid k)=1$. Let $G_{F \mid k}=\operatorname{Aut}(F \mid k)$ be endowed with the compact-open topology for discrete $F$, so that a basis of open neighbourhoods of the identity is given by the subgroups $\operatorname{Aut}(F \mid K)$ with $K \mid k$ a finitely generated subextension of $F \mid k$. For such $K$, we denote the open subgroup $\operatorname{Aut}(F \mid K) \leq G_{F \mid k}$ by $U_{K}$. The group $G_{F \mid k}$ is a Hausdorff and totally disconnected topological group, since $\sigma \in U_{k(x)}$ for all $x \in F$ implies $\sigma=$ id.

In positive characteristic, one has to deal with the phenomenon that purely inseparable extensions are not visible in field automorphism groups. We therefore consider an equivalence relation on the set of subfields of $F$, which we call perfect equivalence. 
Definition 3.1. For a subfield $L$ in $F$, its perfect closure $L^{i}$ in $F$ consists of all elements in $F$ that are purely inseparable over $L$. If char $k=p>0$, it is given by

$$
L^{i}=L^{p^{-\infty}}=\left\{x \in F: x^{p^{n}} \in L \text { for some } n \in \mathbb{N}\right\} .
$$

We call two subfields $L_{1}, L_{2}$ of $F$ perfectly equivalent if $L_{1}^{i}=L_{2}^{i}$.

In characteristic $p>0$, since $p$ th roots are unique, the image of $x \in F$ under an automorphism is uniquely determined by the image of $x^{p^{n}}$, hence $\operatorname{Aut}(F \mid L)=\operatorname{Aut}\left(F \mid L^{i}\right)$ for all subfields $L$ of $F$. Automorphism groups of field extensions are therefore "blind" towards purely inseparable extensions in the sense that perfectly equivalent subfields $L_{1}$ and $L_{2}$ satisfy $\operatorname{Aut}\left(F \mid L_{1}\right)=\operatorname{Aut}\left(F \mid L_{2}\right)$. However, a subfield $L$ of $F$ can be recovered from $\operatorname{Aut}(F \mid L)$ up to perfect equivalence.

Lemma 3.2. Let $F$ be an algebraically closed field. Then for all subfields $L \subseteq F$, we have $F^{\operatorname{Aut}(F \mid L)}=L^{i}$.

Proof. The inclusion $(\supseteq)$ is trivial. If $x \in F$, but $x \notin L^{i}$, there exists some $x^{\prime} \in F \backslash\{x\}$ and an isomorphism $L(x) \cong L\left(x^{\prime}\right)$ over $L$, sending $x$ to $x^{\prime}$; take $x^{\prime}=x+1$ if $x$ is transcendental over $L$, and any root $x^{\prime} \neq x$ of the (not purely inseparable) minimal polynomial of $x$ over $L$ if $x$ is algebraic. Since $F \mid L(x)$ and $F \mid L\left(x^{\prime}\right)$ have equal transcendence degree (possibly infinite), the isomorphism $L(x) \cong L\left(x^{\prime}\right)$ extends to an automorphism $\sigma$ of $F$. We have $\sigma \in \operatorname{Aut}(F \mid L)$, but $\sigma(x) \neq x$, therefore $x \notin F^{\operatorname{Aut}(F \mid L)}$.

Theorem 3.3 (Galois-Type Correspondence). Let $F \mid k$ be a field extension with $F$ algebraically closed and let $G_{F \mid k}=\operatorname{Aut}(F \mid k)$. Then the map $L \mapsto \operatorname{Aut}(F \mid L)$ is injective up to perfect equivalence and restricts to bijections as follows:

$$
\begin{gathered}
\left\{\begin{array}{c}
\text { subfields } L \text { in } F \mid k \text {, up to } \\
\text { perfect equivalence }
\end{array}\right\} \longleftrightarrow\left\{\text { closed subgroups of } G_{F \mid k}\right\} \\
U \mid \\
\left\{\begin{array}{c}
\text { subfields } L \text { in } F \mid k \text { with } \\
\bar{L}=F, \text { up to perfect } \\
\text { equivalence }
\end{array}\right\} \longrightarrow\left\{\begin{array}{c}
\text { compact subgroups of } \\
G_{F \mid k}
\end{array}\right\} \\
U \mid \\
\left\{\begin{array}{c}
\text { finitely generated subfields } \\
L \text { in } F \mid k \text { with } \bar{L}=F, \text { up } \\
\text { to perfect equivalence }
\end{array}\right\} \longrightarrow\left\{\begin{array}{c}
\text { compact open subgroups } \\
\text { of } G_{F \mid k}
\end{array}\right\}
\end{gathered}
$$

Proof. Write $G:=G_{F \mid k}$. For every subfield $L$ in $F \mid k$, the group

$$
\operatorname{Aut}(F \mid L)=\bigcap_{x \in L} U_{k(x)}
$$

is closed in $G$. If $F \mid L$ is algebraic, then the $\operatorname{Aut}(F \mid L)$-orbit of every $x \in F$ is finite and the product $\prod\left(X-x_{i}\right)$ with $x_{i}$ running through this orbit is a separable polynomial annihilating $x$ with coefficients in $F^{\operatorname{Aut}(F \mid L)}=L^{i}$, so that $F$ is separable and hence Galois over $L^{i}$. Thus $\operatorname{Aut}(F \mid L)=\operatorname{Gal}\left(F \mid L^{i}\right)$ is compact if $\bar{L}=F$, and compact open if in addition $L$ is finitely generated over $k$.

Suppose $H$ is a compact subgroup of $G$. Then for every $x \in F$, the orbit $H x$ is compact and discrete, hence finite, so $x$ is a root of a separable polynomial with coefficients in $F^{H}$. Thus $F \mid F^{H}$ is Galois and we have $H=\operatorname{Aut}(F \mid H)$ by Galois theory. This establishes the middle bijection.

It remains to show that if $H \leq G$ is a compact open subgroup, then there exists a finitely generated subfield $K$ in $F \mid k$ with $H=\operatorname{Aut}(F \mid K)$. Since the sets $U_{K}$ with $K \mid k$ finitely generated 
form a neighbourhood basis of the identity, there exists such $K$ with $U_{K} \subseteq H$. Taking fixed fields, we get $F^{H} \subseteq K^{i}$. As $F^{H} \mid F^{H} \cap K$ is purely inseparable and $H$ is compact, we have

$$
\operatorname{Aut}\left(F \mid F^{H} \cap K\right)=\operatorname{Aut}\left(F \mid F^{H}\right)=H,
$$

and $F^{H} \cap K$ is finitely generated over $k$, being contained in the finitely generated extension $K \mid k$.

Remarks. (a) The association $L \mapsto \operatorname{Aut}(F \mid L)$ is compatible with the $G_{F \mid k}$-actions in the sense that

$$
\operatorname{Aut}(F \mid \sigma L)=\sigma \operatorname{Aut}(F \mid L) \sigma^{-1}
$$

for all subextensions $L$ of $F \mid k$ and all $\sigma \in G_{F \mid k}$. Moreover, the $G_{F \mid k^{-}}$action on the set of subextensions of $F \mid k$ is compatible with perfect equivalence since $\sigma(L)^{i}=\sigma\left(L^{i}\right)$.

(b) Let $L$ be a subextension of $F \mid k$ and $H=\operatorname{Aut}(F \mid L)$. Then the normaliser of $H$ in $G_{F \mid k}$ is given by $N_{G_{F \mid k}}(H)=\left\{\sigma \in G_{F \mid k} \mid \sigma L^{i}=L^{i}\right\}$ and the restriction homomorphism $N_{G_{F \mid k}}(H) \rightarrow \operatorname{Aut}\left(L^{i} \mid k\right)$ induces an isomorphism of topological groups

$$
N_{G_{F \mid k}}(H) / H \cong \operatorname{Aut}\left(L^{i} \mid k\right) .
$$

(c) The Galois correspondence is inclusion-reversing in the sense that

$$
L_{1}^{i} \subseteq L_{2}^{i} \Leftrightarrow \operatorname{Aut}\left(F \mid L_{2}\right) \subseteq \operatorname{Aut}\left(F \mid L_{1}\right)
$$

for all subfields $L_{1}$ and $L_{2}$ of $F \mid k$.

(d) If $L_{1} \subseteq L_{2}$ is an algebraic extension of subfields of $F \mid k$, the $\operatorname{index}\left(\operatorname{Aut}\left(F \mid L_{1}\right)\right.$ : $\left.\operatorname{Aut}\left(F \mid L_{2}\right)\right)$ equals the separable degree $\left[L_{2}: L_{1}\right]_{s}$ since the left cosets are in canonical bijection with the $L_{1}$-embeddings $L_{2} \hookrightarrow \overline{L_{1}}$.

(e) If the transcendence degree of $F \mid k$ is finite, then every finitely generated subextension $K$ is contained in a finitely generated extension $L$ with $\bar{L}=F$. Otherwise, there are no subextensions $L$ that are both finitely generated over $k$ and have $\bar{L}=F$. Consequently, $G_{F \mid k}$ is locally compact if and only if $F \mid k$ has finite transcendence degree. If moreover $k$ algebraically closed, the transcendence degree of $F \mid k$ can be recovered: let $U_{K} \leq G_{F \mid k}$ be any compact open subgroup, corresponding to a finitely generated subfield $K \mid k$ with $\bar{K}=F$. Then $\operatorname{trdeg}(F \mid k)=\operatorname{cd}_{\ell}\left(U_{K}\right)$ for all primes $\ell \neq \operatorname{char}(k)$ ([Ser97], Ch. II, Proposition 11).

(f) In general, there exist closed subgroups of $G:=G_{F \mid k}$ that do not arise as $\operatorname{Aut}(F \mid K)$ for some subfield $K$ of $F \mid k$. A subgroup $H \leq G$ arises in this way if and only if the inclusion $H \subseteq \operatorname{Aut}\left(F \mid F^{H}\right)$ is an equality. For a counterexample, consider the closed subgroup $G^{\circ} \subseteq G$ topologically generated by all compact subgroups. If $T$ is a transcendence basis of $F \mid k$, so is $T^{n}:=\left\{t^{n}\right\}_{t \in T}$ for $n \geq 1$. Thus, the groups $\operatorname{Aut}\left(F \mid k\left(T^{n}\right)\right)$ are all compact, hence $F^{G^{\circ}}=\bigcap_{n} k\left(T^{n}\right)^{i}=k^{i}$. However, if $F \mid k$ has finite transcendence degree $\geq 1$ with $x \in F$ transcendental over $k$, then $G^{\circ}$ is a proper subgroup of $G$ as $G^{\circ}$ is unimodular but any automorphism $\sigma \in G$ extending $k(x) \cong k\left(x^{2}\right)$ has Haar modulus $\Delta(\sigma)=2$.

One can show that the subgroups of the form $\operatorname{Aut}(F \mid K) \subseteq G$ are stable under passage to closed supergroups with compact quotient. For subgroups this is false: assuming $1 \leq \operatorname{trdeg}(F \mid k)<\infty$, the Haar modulus induces a surjective homomorphism $\Delta: G / G^{\circ} \rightarrow \mathbb{Q}_{>0}$, yielding many finite index subgroups $H \subsetneq G$ containing $G^{\circ}$. They all satisfy $F^{H}=k^{i}$ and hence $\operatorname{Aut}\left(F \mid F^{H}\right)=G$, so they are not of the form $\operatorname{Aut}(F \mid K)$.

Parts of the Galois-type correspondence appear in the literature as [Jac64], p. 151, Exercise 5; [Shi71], Propositions 6.11 and 6.12; [PŠŠ66], §3, Lemma 1. A statement very close to ours in that it encompasses the case of positive characteristic is contained in [Rov05], Appendix B, 
under the slightly stronger assumptions that $k$ be algebraically closed and that $\operatorname{trdeg}(F \mid k)$ be countable and $\geq 1$.

We now apply the Galois-type correspondence to the situation at hand where $k$ is algebraically closed and $\operatorname{trdeg}(F \mid k)=1$. When we use the term function field in $F$, we shall always mean one of dimension 1 , in other words we exclude the trivial function field $k$. The Galois-type correspondence shows that the function fields $K \mid k$ in $F$ are encoded in $G_{F \mid k}$ as the compact open subgroups $U_{K}$, up to perfect equivalence. By Remark (e) above, the transcendence degree of $F \mid k$ is encoded as the $\ell$-cohomological dimension for $\ell \neq \operatorname{char}(k)$ of any such $U_{K}$.

Proposition 3.4. In the situation of Theorem B, let $\lambda: G_{F \mid k} \stackrel{\sim}{\longrightarrow} G_{F^{\prime} \mid k^{\prime}}$ be an isomorphism. Then also $\operatorname{trdeg}\left(F^{\prime} \mid k^{\prime}\right)=1$ and $\lambda$ induces a bijection

$$
\left\{\begin{array}{c}
\text { function fields } K^{\prime} \mid k^{\prime} \text { in } F^{\prime}, \\
\text { up to perfect equivalence }
\end{array}\right\} \longrightarrow\left\{\begin{array}{c}
\text { function fields } K \mid k \text { in } F \\
\text { up to perfect equivalence }
\end{array}\right\}
$$

given by $K^{\prime} \mapsto K$ whenever $\lambda^{-1}\left(U_{K^{\prime}}\right)=U_{K}$.

We have the an explicit description of perfect equivalence for 1-dimensional function fields.

Proposition 3.5 ([Har77] IV, Proposition 2.5). Let $F \mid k$ be an extension of algebraically closed fields of characteristic $p>0$ and let $K \mid k$ be a 1-dimensional function field in $F$. Then for each $n \in \mathbb{N}_{0}$, the extension $K^{p^{-n}} \mid K$ is the unique purely inseparable extension of $K$ in $F$ of degree $p^{n}$. Moreover, every function field in $F$ perfectly equivalent to $K$ is of the form $K^{p^{n}}$ for some $n \in \mathbb{Z}$. In particular, they form an infinite field tower

$$
\ldots \subset K^{p^{2}} \subset K^{p} \subset K \subset K^{p^{-1}} \subset K^{p^{-2}} \subset \ldots
$$

Recall that for an algebraic field extension $L \mid K$ with relative separable closure $K \subseteq K_{s} \subseteq L$, the inseparable degree of $L \mid K$ is defined as $[L: K]_{i}:=\left[L: K_{s}\right]$.

Definition 3.6. Let $F \mid k$ be an extension of algebraically closed fields and $K_{1}, K_{2}$ one-dimensional function fields in $F$ with $K_{1}^{i} \subseteq K_{2}^{i}$ (but not necessarily $K_{1} \subseteq K_{2}$ ). If $\operatorname{char}(k)=p>0$, we define the generalised inseparable degree $\left[K_{2}: K_{1}\right]_{i} \in p^{\mathbb{Z}}$ to be $p^{-n}\left[K_{2}: K_{1}^{p^{n}}\right]_{i}$ where $n$ is sufficiently large such that $K_{1}^{p^{n}} \subseteq K_{2}$. If $\operatorname{char}(k)=0$, we set $\left[K_{2}: K_{1}\right]_{i}=1$.

We note the following properties of the generalised inseparable degree:

(a) For $K_{1}^{i} \subseteq K_{2}^{i} \subseteq K_{3}^{i}$ one has $\left[K_{3}: K_{1}\right]_{i}=\left[K_{3}: K_{2}\right]_{i}\left[K_{2}: K_{1}\right]_{i}$.

(b) $\quad K_{1} \subseteq K_{2}$ iff $\left[K_{2}: K_{1}\right]_{i} \geq 1$.

(c) $\quad K_{2} \mid K_{1}$ is separable iff $\left[K_{2}: K_{1}\right]_{i}=1$.

For a function field $K \mid k$ in $F$, we can recover the automorphism group $\operatorname{Aut}\left(K^{i} \mid k\right)$ from $\left(G_{F \mid k}, U_{K}\right)$ as the quotient $N_{G_{F \mid k}}\left(U_{K}\right) / U_{K}$ by Remark (b). It is related to $\operatorname{Aut}(K \mid k)$ as follows.

Lemma 3.7. Let $K$ be a one-dimensional function field over an algebraically closed field $k$. Then there is a canonical exact sequence

$$
1 \longrightarrow \operatorname{Aut}(K \mid k) \longrightarrow \operatorname{Aut}\left(K^{i} \mid k\right) \longrightarrow \mathbb{Z} .
$$

Proof. The statement is trivial in characteristic zero, so assume $\operatorname{char}(k)=p>0$. Every automorphism of $K \mid k$ extends uniquely to $K^{i}$, whence the injective homomorphism $\operatorname{Aut}(K \mid k) \hookrightarrow$ $\operatorname{Aut}\left(K^{i} \mid k\right)$. The second map is defined as $\sigma \mapsto \log _{p}[\sigma(K): K]_{i}$ and the exactness is readily checked.

Proof of Theorem $B \Rightarrow$ Theorem A. Consider the diagram

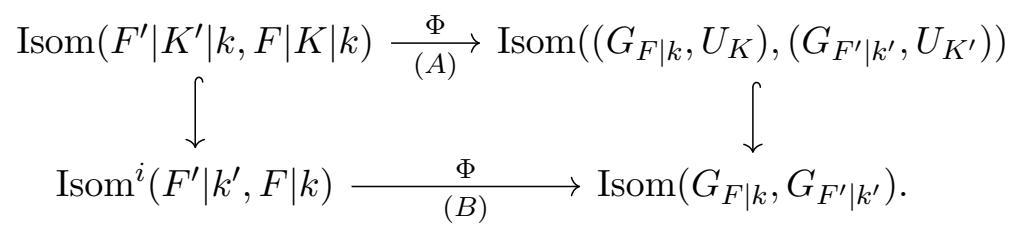


The left vertical map is injective because the condition $\sigma\left(K^{\prime}\right)=K$ determines $\sigma$ uniquely among its Frobenius twists. Theorems A and B assert the bijectivity of the top and bottom horizonal map, respectively. The square is cartesian: For $\sigma \in \operatorname{Isom}\left(F^{\prime}\left|k^{\prime}, F\right| k\right)$ we have $\Phi(\sigma)\left(U_{K}\right)=U_{K^{\prime}}$ if and only if $\sigma\left(K^{\prime}\right)^{i}=K^{i}$ (Galois correspondence), or equivalently if $\tilde{\sigma}\left(K^{\prime}\right)=K$ for some Frobenius twist $\tilde{\sigma} \sim \sigma$.

Proposition 3.8. The map $\Phi$ of Theorem B is injective.

Proof. If $\Phi\left(\sigma_{1}\right)=\Phi\left(\sigma_{2}\right)$, choose an arbitrary function field $K \mid k$ in $F$ and find $K^{\prime}$ such that $\Phi\left(\sigma_{i}\right)\left(U_{K}\right)=U_{K^{\prime}}$ (Galois correspondence). Then in the diagram above, $\sigma_{1}$ and $\sigma_{2}$ come from upstairs where $\Phi$ is injective by Theorem 2.1.

\section{Detecting the Rationality of Function Fields}

The aim of this section is to prove the following Proposition 4.1 and to show that the characteristic $\operatorname{char}(k)$ is encoded in $G_{F \mid k}$ (Proposition 4.5).

Proposition 4.1. Given $\lambda: G_{F \mid k} \stackrel{\sim}{\longrightarrow} G_{F^{\prime} \mid k^{\prime}}$, the bijection of Proposition 3.4 maps rational function fields to rational function fields.

Note that the rationality of a function field depends only on its perfect equivalence class, for the function fields perfectly equivalent to $k(x)$ are given by $k(x)^{p^{n}}=k\left(x^{p^{n}}\right)$ for $n \in \mathbb{Z}$, where $\operatorname{char}(k)=p>0$. Our proof of Proposition 4.1 is an adaption of [Rov03], Lemma 3.1 (1) that takes into account the possibility of positive characteristic.

Definition 4.2. Let $G$ be a group and $n \in \mathbb{N}$. An element $x \in G$ is called $n$-divisible if $y^{n}=x$ for some $y \in G$. It is called infinitely $n$-divisible if there exists a sequence $\left(x_{1}, x_{2}, \ldots\right)$ with $x_{1}=x$ and $x_{i}=x_{i+1}^{n}$ for all $i \in \mathbb{N}$. We say the group $G$ is $n$-divisible if every element is so.

Recall that a group is virtually abelian if it contains an abelian subgroup of finite index.

Lemma 4.3. Let $k$ be an algebraically closed field. Then $\operatorname{Aut}\left(\mathbb{P}_{k}^{1}\right)$ is not virtually abelian, it is $\ell$-divisible for every prime number $\ell \neq \operatorname{char}(k)$ but not $p$-divisible if $\operatorname{char}(k)=p>0$.

Proof. Suppose $H \leq \operatorname{Aut}\left(\mathbb{P}_{k}^{1}\right)$ has finite index. Then $H$ contains a nontrivial translation $\phi(z)=$ $z+b, b \neq 0$, and a nontrivial homothety $\psi(z)=a z, a \neq 1$. They do not commute since

$$
\begin{aligned}
& \psi \circ \phi(z)=a z+a b, \\
& \phi \circ \psi(z)=a z+b,
\end{aligned}
$$

but $a b \neq b$. It is enough to show the $\ell$-divisibility of $\operatorname{GL}(2, k)$ as this property descends to the quotient $\operatorname{Aut}\left(\mathbb{P}_{k}^{1}\right)=\operatorname{PGL}(2, k)$. Given $A \in \mathrm{GL}(2, k)$, we may assume it is in Jordan normal form

$$
A=\left(\begin{array}{cc}
\lambda & 0 \\
0 & \mu
\end{array}\right) \quad \text { or } \quad A=\left(\begin{array}{cc}
\lambda & 1 \\
0 & \lambda
\end{array}\right) .
$$

Then a matrix $B \in \mathrm{GL}(2, k)$ with $B^{\ell}=A$ is given by

$$
B=\left(\begin{array}{cc}
\lambda^{1 / \ell} & 0 \\
0 & \mu^{1 / \ell}
\end{array}\right) \quad \text { or } \quad B=\lambda^{1 / \ell}\left(\begin{array}{cc}
1 & \lambda^{-1} / \ell \\
0 & 1
\end{array}\right),
$$

respectively. Suppose $\operatorname{char}(k)=p>0$ and assume for contradiction that there exists $\psi \in$ $\operatorname{Aut}\left(\mathbb{P}_{k}^{1}\right)$ such that $\psi^{p}(z)=z+1$. If $P \in \mathbb{P}_{k}^{1}$ is a fixed point of $\psi$, it is also a fixed point of $\psi^{p}$, hence $P=\infty$. As a Möbius transformation with $\infty$ as its only fixed point, $\psi$ is a translation, $\psi(z)=z+a$ for some $a \in k$. But then $\psi^{p}(z)=z+p a=z \neq z+1$, contradiction!

Lemma 4.4. Let $K \mid k$ be a 1-dimensional function field with perfect closure $K^{i}$ and let $\ell \neq$ char $(k)$ be a prime number. Then $K$ is rational if and only if the subgroup of $\operatorname{Aut}\left(K^{i} \mid k\right)$ generated by the infinitely $\ell$-divisible elements is not virtually abelian. 
Proof. By Lemma 3.7, the infinitely $\ell$-divisible elements of Aut $\left(K^{i} \mid k\right)$ are in fact infinitely $\ell$ divisible elements in the subgroup $\operatorname{Aut}(K \mid k)$. Moreover, if $C$ is a complete nonsingular model of $K \mid k$, we have isomorphisms

$$
\operatorname{Aut}(K \mid k) \cong \operatorname{Aut}(C)^{\mathrm{op}} \cong \operatorname{Aut}(C) .
$$

Thus, we define $H$ as the subgroup of $\operatorname{Aut}(C)$ generated by the infinitely $\ell$-divisible elements and show that $C$ is rational if and only if $H$ is not virtually abelian.

If $C \cong \mathbb{P}^{1}$ is rational, we have $H=\operatorname{Aut}(C) \cong \operatorname{Aut}\left(\mathbb{P}_{k}^{1}\right)$ and this is not virtually abelian by Lemma 4.3. If $C$ has genus 1 , it is a principal homogeneous space under the elliptic curve $E=\operatorname{Jac} C$ and we have $\operatorname{Aut}(C) \cong E(k) \rtimes \operatorname{Aut}(E)$. The abelian subgroup $E(k)$ is divisible, hence contained in $H$, so that $H$ is virtually abelian, $\operatorname{Aut}(E)$ being finite. For $C$ of higher genus, $\operatorname{Aut}(C)$ is finite and $H$ is virtually abelian via the trivial subgroup.

Proof of Proposition 4.1. Let $K \mid k$ and $K^{\prime} \mid k^{\prime}$ be function fields with $\lambda^{-1}\left(U_{K^{\prime}}\right)=U_{K}$. Then $\lambda$ induces an isomorphism

$$
N_{G_{F \mid k}}\left(U_{K}\right) / U_{K} \stackrel{\sim}{\longrightarrow} N_{G_{F^{\prime} \mid k^{\prime}}}\left(U_{K^{\prime}}\right) / U_{K^{\prime}},
$$

hence $\operatorname{Aut}\left(K^{i} \mid k\right) \cong \operatorname{Aut}\left(K^{i} \mid k^{\prime}\right)$. Now use Lemma 4.4 with $\ell$ a prime number $\neq \operatorname{char}(k), \operatorname{char}\left(k^{\prime}\right)$ to test the rationality of $K$ (resp. $K^{\prime}$ ) by means of these automorphism groups.

Proposition 4.5. For $F \mid k$ and $F^{\prime} \mid k^{\prime}$ as in Theorem $B$, if $G_{F \mid k} \cong G_{F^{\prime} \mid k^{\prime}}$, then $\operatorname{char}(k)=\operatorname{char}\left(k^{\prime}\right)$.

Proof. Choose an arbitrary rational function field $K \mid k$ in $F$ and $K^{\prime}$ with $\lambda^{-1}\left(U_{K^{\prime}}\right)=U_{K}$. Then $\operatorname{Aut}\left(K^{i} \mid k\right) \cong \operatorname{Aut}\left(K^{\prime i} \mid k^{\prime}\right)$ as above and passing to the subgroups generated by the infinitely $\ell$ divisible elements, $\operatorname{Aut}\left(\mathbb{P}_{k}^{1}\right) \cong \operatorname{Aut}\left(\mathbb{P}_{k^{\prime}}^{1}\right)$. By Lemma 4.3, $\operatorname{char}(k)$ is the unique prime $p$ for which $\operatorname{Aut}\left(\mathbb{P}_{k}^{1}\right)$ is not $p$-divisible, or zero if no such prime exists, so it is the same for $k$ and $k^{\prime}$.

\section{Detecting Decomposition Groups}

Our next aim is to give a group-theoretic characterisation in terms of $\left(G_{F \mid k}, U_{K}\right)$ of the decomposition groups in the pro- $\ell$ abelian Galois group $U_{K}^{\mathrm{ab}, \ell}$ (Proposition 5.4). We start by recollecting some generalities. Let $k$ be an algebraically closed field, $\ell \neq \operatorname{char}(k)$ a fixed prime number and $K \mid k$ the function field of a complete nonsingular curve $C$ over $k$. The normalised discrete valuations on $K \mid k$ correspond bijectively to closed points of $C$ and we write $\operatorname{ord}_{P}$ for the valuation associated with $P \in C(k)$. Denote by $K^{\text {ab }, \ell}$ the maximal pro- $\ell$ abelian extension of $K$, obtained by adjoining the $\ell^{n}$ th roots of all elements of $K$ for all $n \in \mathbb{N}$. The Galois group $\operatorname{Gal}\left(K^{\mathrm{ab}, \ell} \mid K\right)$ acts transitively on the set of valuations of $K^{\mathrm{ab}, \ell}$ extending $\operatorname{ord}_{P}$ and their common stabiliser is the (pro- $\ell$ ) decomposition group of $P$, which we denote by $Z_{P}$. Let $Z_{\text {tot }}(C) \subseteq \operatorname{Gal}\left(K^{\mathrm{ab}, \ell} \mid K\right)$ be the closed subgroup topologically generated by all $Z_{P}$, called the (pro- $\ell$ ) total decomposition group of $K \mid k$. Its fixed field is the maximal completely split pro- $\ell$ abelian extension of $K$, or equivalently the maximal unramified pro- $\ell$ abelian extension since all residue field extensions are trivial. Thus, we have an exact sequence

$$
1 \longrightarrow Z_{\mathrm{tot}}(C) \longrightarrow \operatorname{Gal}\left(K^{\mathrm{ab}, \ell} \mid K\right) \longrightarrow \pi_{1}^{\mathrm{ab}, \ell}(C) \longrightarrow 1
$$

where $\pi_{1}^{\mathrm{ab}, \ell}(C)$ is the pro- $\ell$ abelianisation of the algebraic fundamental group of $C$.

Denote by $\mu_{\ell^{n}}(k)$ the group of $\ell^{n}$ th roots of unity in $k$ and by $\mathbb{Z}_{\ell}(1)=\lim _{n} \mu_{\ell^{n}}(k)$ the $\ell$-adic Tate module of $k^{\times}$. By Kummer theory, there is a natural isomorphism of topological groups

$$
\operatorname{Gal}\left(K^{\mathrm{ab}, \ell} \mid K\right) \cong \operatorname{Hom}\left(K^{\times}, \mathbb{Z}_{\ell}(1)\right) .
$$

Definition 5.1. Write $\operatorname{Map}\left(C, \mathbb{Z}_{\ell}(1)\right) / \mathbb{Z}_{\ell}(1)$ for the group of set-theoretic functions $C(k) \rightarrow$ $\mathbb{Z}_{\ell}(1)$ modulo the subgroup of constant functions. A function $f: C(k) \rightarrow \mathbb{Z}_{\ell}(1)$ is a 1-point function at $P \in C(k)$ if it is constant on $C(k) \backslash\{P\}$. 
Our 1-point functions are called " $\delta$-functions" in [Rov03]. Note that the 1-point functions at $P$ are closed under addition of constants, so the notion makes sense even for elements of $\operatorname{Map}\left(C, \mathbb{Z}_{\ell}(1)\right) / \mathbb{Z}_{\ell}(1)$. A typical 1-point function at $P$ has the form $\delta_{P} \omega$ with $\omega \in \mathbb{Z}_{\ell}(1)$, where $\delta_{P}: C(k) \rightarrow\{0,1\}$ is the Kronecker delta function. We recall the following well-known description of decomposition groups.

Proposition 5.2. There are canonical isomorphisms

(a) $Z_{P} \cong \mathbb{Z}_{\ell}(1)$, for all closed points $P \in C$,

(b) $Z_{\text {tot }}(C) \cong \operatorname{Map}\left(C, \mathbb{Z}_{\ell}(1)\right) / \mathbb{Z}_{\ell}(1) \cong \operatorname{Hom}\left(\operatorname{Div}^{0}(C), \mathbb{Z}_{\ell}(1)\right)$,

under which the inclusion $Z_{P} \subseteq Z_{\text {tot }}(C)$ is isomorphic to $\mathbb{Z}_{\ell}(1) \stackrel{\delta_{P}}{\longrightarrow} \operatorname{Map}\left(C, \mathbb{Z}_{\ell}(1)\right) / \mathbb{Z}_{\ell}(1)$ with image the 1-point functions at $P$.

Proof. Let $K_{P}$ be the henselisation of $K$ with respect to $\operatorname{ord}_{P}$, let $\mathcal{O}_{C, P}^{h}$ be its valuation ring and $K_{P}^{\mathrm{ab}, \ell}$ its maximal pro- $\ell$ abelian extension, which is also the henselisation of $K^{\mathrm{ab}, \ell}$ with respect to a valuation extending ord ${ }_{P}$. The inclusion $Z_{P} \subseteq \operatorname{Gal}\left(K^{\mathrm{ab}, \ell} \mid K\right)$ is isomorphic to $\operatorname{Hom}\left(K_{P}^{\times}, \mathbb{Z}_{\ell}(1)\right) \hookrightarrow \operatorname{Hom}\left(K^{\times}, \mathbb{Z}_{\ell}(1)\right)$ by functoriality of Kummer theory. We have a split exact sequence

$$
1 \longrightarrow \mathcal{O}_{C, P}^{h, \times} \longrightarrow K_{P}^{\times} \underset{\operatorname{ord}_{P}}{\longleftrightarrow} \mathbb{Z} \longrightarrow 1
$$

and obtain another short exact sequence upon taking $\operatorname{Hom}\left(-, \mathbb{Z}_{\ell}(1)\right)$. The group $\mathcal{O}_{C, P}^{h, \times}$ is $\ell$ divisible by Hensel's lemma, hence $\operatorname{Hom}\left(\mathcal{O}_{C, P}^{h, \times}, \mathbb{Z}_{\ell}(1)\right)=0$ and we get the canonical isomorphism

$$
\mathbb{Z}_{\ell}(1) \cong \operatorname{Hom}\left(K_{P}^{\times}, \mathbb{Z}_{\ell}(1)\right)
$$

given by $\omega \mapsto\left[x \mapsto \operatorname{ord}_{P}(x) \omega\right]$. Consider the exact sequence

$$
K^{\times} \stackrel{\text { div }}{\longrightarrow} \operatorname{Div}^{0}(C) \longrightarrow \operatorname{Pic}^{0}(C) \longrightarrow 1 .
$$

We have $\operatorname{Hom}\left(\operatorname{Pic}^{0}(C), \mathbb{Z}_{\ell}(1)\right)=0$ as $\operatorname{Pic}^{0}(C)$ is $\ell$-divisible, hence

$$
\operatorname{div}^{*}: \operatorname{Hom}\left(\operatorname{Div}^{0}(C), \mathbb{Z}_{\ell}(1)\right) \longleftrightarrow \operatorname{Hom}\left(K^{\times}, \mathbb{Z}_{\ell}(1)\right) .
$$

is injective. The groups are endowed with the compact-open topology and div* is a topological embedding as it is continuous and the groups are compact Hausdorff. We have an isomorphism

$$
\operatorname{Hom}\left(\operatorname{Div}^{0}(C), \mathbb{Z}_{\ell}(1)\right) \cong \operatorname{Map}\left(C, \mathbb{Z}_{\ell}(1)\right) / \mathbb{Z}_{\ell}(1)
$$

induced by $\operatorname{Hom}\left(\operatorname{Div}(C), \mathbb{Z}_{\ell}(1)\right) \cong \operatorname{Map}\left(C, \mathbb{Z}_{\ell}(1)\right)$. The maps

$$
\mathbb{Z}_{\ell}(1) \cong \operatorname{Hom}\left(K_{P}^{\times}, \mathbb{Z}_{\ell}(1)\right) \hookrightarrow \operatorname{Hom}\left(K^{\times}, \mathbb{Z}_{\ell}(1)\right)
$$

all factor through $\mathbb{Z}_{\ell}(1) \stackrel{\delta_{P} \cdot}{\longrightarrow} \operatorname{Map}\left(C, \mathbb{Z}_{\ell}(1)\right) / \mathbb{Z}_{\ell}(1)$ and (b) follows since the images generate $\operatorname{Map}\left(C, \mathbb{Z}_{\ell}(1)\right) / \mathbb{Z}_{\ell}(1)$ topologically.

We are interested in the functorial behaviour of decomposition groups. Let $\phi: C_{2} \rightarrow C_{1}$ be a dominant morphism between complete nonsingular curves over $k$ and $\phi^{*}: K_{1} \hookrightarrow K_{2}$ the corresponding function field extension. Embed $K_{1}$ and $K_{2}$ in a common algebraic closure $F$ and let $U_{K_{i}}=\operatorname{Aut}\left(F \mid K_{i}\right)$. The inclusion $U_{K_{2}} \subseteq U_{K_{1}}$ induces two homomorphisms in opposite directions between the pro- $\ell$ abelianisations:

- the corestriction cor : $U_{K_{2}}^{\mathrm{ab}, \ell} \rightarrow U_{K_{1}}^{\mathrm{ab}, \ell}$; and

- the restriction res : $U_{K_{1}}^{\mathrm{ab}, \ell} \rightarrow U_{K_{2}}^{\mathrm{ab}, \ell}$, also called the transfer tr.

They come from profinite group homology via the isomorphisms $U_{K_{i}}^{\mathrm{ab}, \ell} \cong H_{1}\left(U_{K_{i}}, \mathbb{Z}_{\ell}\right)$. The corestriction is also the natural map from the functoriality of $(-)^{\mathrm{ab}, \ell}$. Their effect on decomposition groups can be expressed via the pullback and pushward map of divisors. 
Proposition 5.3. The following squares commute:
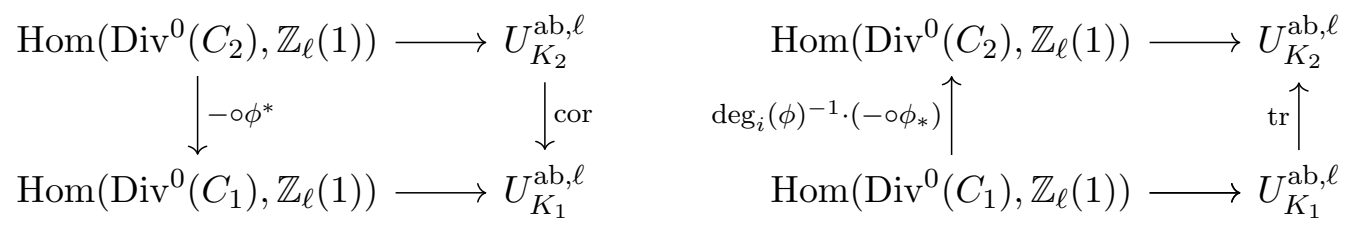

Here $\operatorname{deg}_{i}(\phi)=\left[K_{2}: K_{1}\right]_{i}$ denotes the inseparable degree, which is either 1 or a power of the characteristic of $k$ so that multiplication by $\operatorname{deg}_{i}(\phi)^{-1}$ on $\mathbb{Z}_{\ell}(1)$ is well-defined.

Proof. From Kummer theory and the fact that corestriction is Pontryagin dual to restriction we have two commutative squares.

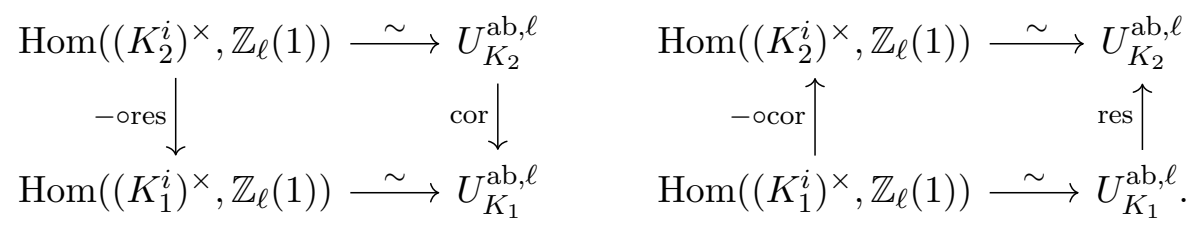

The restriction res : $\left(K_{1}^{i}\right)^{\times} \rightarrow\left(K_{2}^{i}\right)^{\times}$is the extension of the inclusion $\phi^{*}: K_{1}^{\times} \hookrightarrow K_{2}^{\times}$and the corestriction cor : $\left(K_{2}^{i}\right)^{\times} \rightarrow\left(K_{1}^{i}\right)^{\times}$is the group-theoretic norm

$$
\operatorname{cor}\left(x_{2}\right)=\prod_{\sigma \in U_{K_{1}} / U_{K_{2}}} \sigma x_{2},
$$

which on $K_{2}^{\times}$is related to the field-theoretic norm $\mathrm{Nm}: K_{2}^{\times} \rightarrow K_{1}^{\times}$by $\operatorname{cor}\left(x_{2}\right)=\operatorname{Nm}\left(x_{2}\right)^{\operatorname{deg}_{i}(\phi)}$. The claim follows now from the formulae for the divisor map

$$
\begin{aligned}
\operatorname{div}\left(\phi^{*} x_{1}\right) & =\phi^{*}\left(\operatorname{div}\left(x_{1}\right)\right), \\
\operatorname{div}\left(\operatorname{Nm} x_{2}\right) & =\phi_{*}\left(\operatorname{div}\left(x_{2}\right)\right) .
\end{aligned}
$$

Given $\phi: C_{2} \rightarrow C_{1}$ as above, we use the terms corestriction and transfer not just for the homomorphisms between the pro- $\ell$ abelianised absolute Galois groups but also for the corresponding maps between the groups $\operatorname{Hom}\left(\operatorname{Div}^{0}\left(C_{i}\right), \mathbb{Z}_{\ell}(1)\right)$. On 1-point functions and hence on decomposition groups they act as follows. Given $P \in C_{2}(k), Q \in C_{1}(k)$ and $\omega \in \mathbb{Z}_{\ell}(1)$, we have

$$
\begin{aligned}
\operatorname{cor}\left(\delta_{P} \omega\right) & =e_{\phi}(P) \delta_{\phi(P)} \omega, \\
\operatorname{tr}\left(\delta_{Q} \omega\right) & =\operatorname{deg}_{i}(\phi)^{-1} \mathbb{1}_{\phi^{-1}(Q)} \omega,
\end{aligned}
$$

where $e_{\phi}(P)$ denotes the ramification index of $\phi$ at $P$ and $\mathbb{1}_{\phi^{-1}(Q)}$ denotes the characteristic function of $\phi^{-1}(Q)$ on $C_{2}(k)$.

With these generalities at hand, we turn to the proof of the following.

Proposition 5.4. In the situation of Theorem B, given $\lambda: G_{F \mid k} \cong G_{F^{\prime} \mid k^{\prime}}$, let $K \mid k$ and $K^{\prime} \mid k^{\prime}$ be corresponding function fields with complete nonsingular models $C, C^{\prime}$. Then the isomorphism $\lambda^{\mathrm{ab}, \ell}: U_{K}^{\mathrm{ab}, \ell} \stackrel{\sim}{\longrightarrow} U_{K^{\prime}}^{\mathrm{ab}, \ell}$ maps decomposition groups to decomposition groups, inducing a bijection

$$
\lambda^{*}: C^{\prime}\left(k^{\prime}\right) \stackrel{\sim}{\longrightarrow} C(k) .
$$

We first treat the case of rational function fields and then extend to the general case. Let $K \mid k$ be a rational function field in $F$ with complete nonsingular model $C$. Let $H \subseteq N_{G_{F \mid k}}\left(U_{K}\right) / U_{K}$ be the subgroup generated by the infinitely $\ell$-divisible elements. Recall from the proof of Lemma 4.4 the isomorphism $H \cong \operatorname{Aut}(K \mid k) \cong \operatorname{Aut}(C)^{\mathrm{op}}$. For $\sigma \in H$, denote by $\sigma^{*}$ the corresponding automorphism of $C$ and by $\operatorname{ad}(\sigma)$ the automorphism of $U_{K}^{\text {ab, } \ell}$ induced by conjugation. For $P \in C(k)$, denote by $\operatorname{Stab}_{H}(P)$ the stabiliser

$$
\operatorname{Stab}_{H}(P)=\left\{\sigma \in H: \sigma^{*}(P)=P\right\} .
$$


Lemma 5.5. An element of $U_{K}^{\mathrm{ab}, \ell}$ belongs to the decomposition subgroup $Z_{P}$ if and only if it is fixed by $\operatorname{ad}(\sigma)$ for all $\sigma \in \operatorname{Stab}_{H}(P)$.

Proof. Since the homomorphism $\operatorname{Map}\left(C, \mathbb{Z}_{\ell}(1)\right) / \mathbb{Z}_{\ell}(1) \rightarrow U_{K}^{\mathrm{ab}, \ell}$ from Proposition 5.2 depends functorially on $F|K| k$ with respect to isomorphisms, any $\sigma \in H$ induces a commutative square

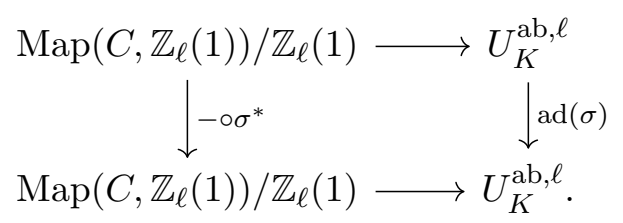

By the exact sequence 5.1, the horizontal maps are injective with cokernel $\pi_{1}^{\mathrm{ab}, \ell}(C)=1$, so they are in fact isomorphisms. We have to show that $f: C(k) \rightarrow \mathbb{Z}_{\ell}(1)$ is a 1-point function at $P$ if and only if $f \circ \sigma^{*} \equiv f \bmod$ constants for all $\sigma \in \operatorname{Stab}_{H}(P)$. As these $\sigma^{*}$ fix $P$, the congruence mod constants amounts to equality. Now $\operatorname{Stab}_{H}(P)$ acts transitively on $C(k) \backslash\{P\}$, therefore if $f \circ \sigma^{*}=f$ for all $\sigma \in \operatorname{Stab}_{H}(P)$, then $f$ is constant on $C(k) \backslash\{P\}$.

We are led to the question of a group-theoretic characterisation of the stabiliser subgroups in Aut $\left(\mathbb{P}^{1}\right)$. Recall that an automorphism of $\mathbb{P}^{1}$ is parabolic if it has a single fixed point. E. g., the parabolic elements with fixed point $\infty$ are the non-trivial translations $z \mapsto z+b$ with $b \in k$.

Lemma 5.6. (a) An element of $\operatorname{Aut}\left(\mathbb{P}^{1}\right)$ is parabolic if and only if it is uniquely $\ell$-divisible. (b) A subgroup of $\operatorname{Aut}\left(\mathbb{P}^{1}\right)$ is the stabiliser subgroup of a point $P \in \mathbb{P}^{1}(k)$ if and only if it is the normaliser of the centraliser of a parabolic element.

Proof. The parabolic elements constitute the conjugacy class of the translation $z+1$. This is uniquely $\ell$-divisible with $z+1 / \ell$ as the unique dividing element. Indeed, if $\varphi^{\ell}=z+1$, then $\varphi$ commutes with $z+1$, which implies that $\varphi$ is also parabolic with fixed point $\infty$, i.e. a translation, necessarily equal to $\varphi(z)=z+1 / \ell$. The non-parabolic elements are conjugate to a homothety $a z$ and are non-uniquely $\ell$-divisible as there are $\ell$ distinct roots $\sqrt[\ell]{a}$.

For (b) it suffices to show that the stabiliser of $\infty$, which consists of the affine transformations $a z+b$ with $a \in k^{\times}$and $b \in k$, is the normaliser of the centraliser of $z+1$. The centraliser of $z+1$ is the group of translations $z+b$. These are indeed normalised by the affine transformations and conversely, if $\psi \circ(z+1) \circ \psi^{-1}$ is a translation, we have $\psi(\infty)=\infty$ since $\psi$ maps the fixed point of $z+1$ to that of $\psi \circ(z+1) \circ \psi^{-1}$.

The two preceding lemmas prove Proposition 5.4 in the rational case. For the general case, call a compact open subgroup $V \subseteq G_{F \mid k}$ rational if $V=U_{k(x)}$ for some rational function field $k(x)$ in $F$. Recall that the rational compact open subgroups are group-theoretically distinguished by Proposition 4.1.

Lemma 5.7. Let $K \mid k$ be a function field in $F$ with complete nonsingular model $C$. A subgroup $Z \subseteq U_{K}^{\mathrm{ab}, \ell}$ is the decomposition group of a point $P \in C(k)$ if and only if the following hold:

(a) There exists a rational $V \supseteq U_{K}$ such that $Z$ is the image of a decomposition group under the transfer map $\operatorname{tr}: V^{\mathrm{ab}, \ell} \rightarrow U_{K}^{\mathrm{ab}, \ell}$.

(b) For every rational $V \supseteq U_{K}$, the image of $Z$ under the corestriction map cor : $U_{K}^{\mathrm{ab}, \ell} \rightarrow$ $V^{\mathrm{ab}, \ell}$ is contained in a decomposition group.

Proof. Assume that $Z=Z_{P}$ is a decomposition group. By Riemann-Roch, there exists a morphism $\phi: C \rightarrow \mathbb{P}^{1}$ having a pole at $P$ and no other poles. Let $k(x) \subseteq K$ be the corresponding inclusion of function fields in $F$ and $V=U_{k(x)} \supseteq U_{K}$. For a 1-point function $\delta_{\infty} \omega$ at $\infty \in \mathbb{P}^{1}$, we have

$$
\operatorname{tr}\left(\delta_{\infty} \omega\right)=\operatorname{deg}_{i}(\phi)^{-1} \mathbb{1}_{\phi^{-1}(\infty)} \omega=\operatorname{deg}_{i}(\phi)^{-1} \delta_{P} \omega
$$


which shows $\operatorname{tr}\left(Z_{\infty}\right)=Z_{P}$. For (b), let $V \subseteq U_{K}$ be any rational group and $\phi: C \rightarrow \mathbb{P}^{1}$ a corresponding morphism. Then for $P \in C(k)$, we have

$$
\operatorname{cor}\left(\delta_{P} \omega\right)=e_{\phi}(P) \delta_{\phi(P)} \omega,
$$

hence $\operatorname{cor}\left(Z_{P}\right)=e_{\phi}(P) Z_{\phi(P)}$ is contained in the decomposition group $Z_{\phi(P)}$.

Suppose conversely that $Z \subseteq U_{K}^{\mathrm{ab}, \ell}$ satisfies the two conditions. The transfer calculation above shows that (a) is equivalent to the existence of a non-empty finite subset $S \subseteq C(k)$ (namely a fibre of a morphism $C \rightarrow \mathbb{P}^{1}$ ) such that the elements of $Z$ correspond to the functions $f: C(k) \rightarrow \mathbb{Z}_{\ell}(1)$ that are constant on $S$ and vanish on $C(k) \backslash S$. Let $\phi: C \rightarrow \mathbb{P}^{1}$ be a morphism separating the points in $S$ and let $V \supseteq U_{K}$ be the corresponding rational group. For $f=\mathbb{1}_{S} \omega$ we have

$$
\operatorname{cor}(f)(Q)=\left(f \circ \phi^{*}\right)(Q)= \begin{cases}e_{\phi}(P) \omega & \text { if } \phi^{-1}(Q) \cap S=\{P\}, \\ 0 & \text { if } \phi^{-1}(Q) \cap S=\emptyset .\end{cases}
$$

By (b), this should be a 1-point function at a point, hence $S$ consists just of a single point, $S=\{P\}$, and $Z=Z_{P}$ is the decomposition group of $P$.

\section{Proof of Main Theorem}

Lemma 6.1. In the situation of Theorem $B$, given $\lambda: G_{F \mid k} \stackrel{\sim}{\longrightarrow} G_{F^{\prime} \mid k^{\prime}}$, let $K_{1} \subseteq K_{2}$ and $K_{1}^{\prime} \subseteq K_{2}^{\prime}$ be extensions of function fields with $\lambda^{-1}\left(U_{K_{i}^{\prime}}\right)=U_{K_{i}}$, corresponding to morphisms $\phi: C_{2} \rightarrow C_{1}$ and $\phi^{\prime}: C_{2}^{\prime} \rightarrow C_{1}^{\prime}$. Let $\lambda^{*}: \operatorname{Div}\left(C_{i}^{\prime}\right) \rightarrow \operatorname{Div}\left(C_{i}\right)$ be the linear extension of the bijection from Proposition 5.4. Of the two squares

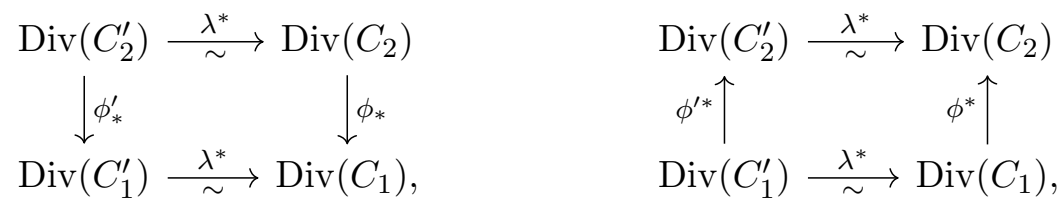

the first always commutes and the second commutes if $\operatorname{deg}_{i}(\phi)=\operatorname{deg}_{i}\left(\phi^{\prime}\right)$.

Proof. By equations (5.2) and (5.3), the corestriction and transfer maps restrict for $Q \in C_{1}$ as follows

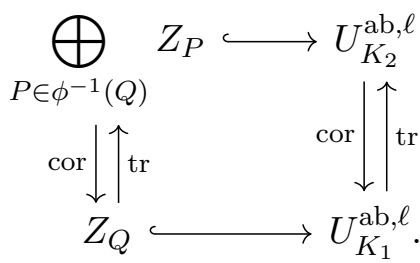

For $P \in \phi^{-1}(Q)$, we have $\operatorname{cor}\left(Z_{P}\right)=e_{\phi}(P) Z_{Q} \subseteq Z_{Q}$ of finite index. This determines $Q=$ $\phi(P)$ uniquely since decomposition groups of different points have trivial intersection, hence the commutativity of the first square. We have

$$
(\operatorname{tr} \circ \operatorname{cor})\left(\delta_{P} \omega\right)=\frac{e_{\phi}(P)}{\operatorname{deg}_{i}(\phi)} \mathbb{1}_{\phi^{-1}(Q)} \omega=\frac{e_{\phi}(P)}{\operatorname{deg}_{i}(\phi)} \sum_{P^{\prime} \in \phi^{-1}(Q)} \delta_{P^{\prime}} \omega,
$$

thus the endomorphism of $Z_{P}$ induced by trocor is multiplication by $e_{\phi}(P) / \operatorname{deg}_{i}(\phi)$. Therefore, assuming equal inseparable degrees of $\phi$ and $\phi^{\prime}$, the ramification indices match and the second square commutes.

Remark 6.2. The $\ell$-part of the ramification index $e_{\phi}(P)$ can also be reconstructed as the index $\left(Z_{Q}: \operatorname{cor}\left(Z_{P}\right)\right)$. If one restricts oneself to fields of characteristic zero, $\ell$ can be an arbitrary prime and this would give an alternative reconstruction of ramification indices. 
The projectivisation of a $k$-vector space $V$ is the set $\mathbb{P}_{k} V=(V \backslash\{0\}) / k^{\times}$, together with the projective lines as distinguished subsets. A map $\mathbb{P}_{k^{\prime}} V^{\prime} \hookrightarrow \mathbb{P}_{k} V$ is a projective embedding if it is injective and maps lines onto lines. It is a collineation if it admits an inverse projective embedding. Given a function field $K \mid k$ with complete nonsingular model $C$, we view $K^{\times} / k^{\times}=$ $\mathbb{P}_{k} K$ as a projective space over $k$, and with it the group $\operatorname{PDiv}(C) \cong K^{\times} / k^{\times}$of principal divisors. The strategy is then to recover the projective structure on $\operatorname{PDiv}(C)$ and reconstruct the function field $K \mid k$ by an application of the fundamental theorem of projective geometry. This idea appears already in [Bog91] and later in [BT08] (Theorem 3.6) and [Pop12]. We shall use the following slightly more general form for projective embeddings rather than collineations.

Theorem 6.3 (Fundamental Theorem of Projective Geometry, [Art57], Thm II.2.26). Let $k$ and $k^{\prime}$ be arbitrary fields, let $V$ and $V^{\prime}$ be vector spaces of dimension $\geq 3$ over $k$ and $k^{\prime}$, respectively, and let $\varphi: \mathbb{P}_{k^{\prime}} V^{\prime} \longleftrightarrow \mathbb{P}_{k} V$ be a projective embedding. Then there exist a field isomorphism $\tau: k^{\prime} \stackrel{\sim}{\longrightarrow} k$ and $a \tau$-semilinear injection $\Phi: V^{\prime} \hookrightarrow V$ lifting $\varphi$, i.e.

$$
\varphi\left(v^{\prime} \bmod k^{\prime \times}\right)=\Phi\left(v^{\prime}\right) \bmod k^{\times} \quad \text { for } v^{\prime} \in V^{\prime} \backslash\{0\} .
$$

If $(\tilde{\tau}, \tilde{\Phi})$ is another such pair, then $\tilde{\tau}=\tau$ and $\tilde{\Phi}=m_{\alpha} \circ \Phi$ for a unique $\alpha \in k^{\times}$, where $m_{\alpha} \in \operatorname{Aut}_{k}(V)$ is multiplication by $\alpha$.

Lemma 6.4. Let $k$ and $k^{\prime}$ be arbitrary fields, $K \mid k$ and $K^{\prime} \mid k^{\prime}$ two field extensions of degree $\geq 3$ and suppose that

$$
\varphi: K^{\prime \times} / k^{\prime \times} \hookrightarrow K^{\times} / k^{\times}
$$

is simultaneously a projective embedding and a homomorphism of abelian groups. Then there exists a unique homomorphism of field extensions

$$
\Phi: K^{\prime}\left|k^{\prime} \longleftrightarrow K\right| k
$$

lifting $\varphi$ and restricting to an isomorphism $k^{\prime} \cong k$.

Proof. Let $(\tau, \Phi)$ be the pair from the fundamental theorem of projective geometry, which is uniquely determined by requiring $\Phi(1)=1$. We have to show that $\Phi$ respects multiplication. Fix $x^{\prime} \in K^{\prime x}$ and let $m_{x^{\prime}}: K^{\prime} \rightarrow K^{\prime}$ and $m_{\Phi\left(x^{\prime}\right)}: K \rightarrow K$ be multiplication by $x^{\prime}$ and $\Phi\left(x^{\prime}\right)$, respectively. We need to show that the two maps

$$
\Phi \circ m_{x^{\prime}} \text { and } m_{\Phi\left(x^{\prime}\right)} \circ \Phi: K^{\prime} \rightarrow K
$$

are equal. They are both $\tau$-semilinear and by multiplicativity of $\varphi$ induce the same projective embedding $K^{\prime \times} / k^{\prime \times} \rightarrow K^{\times} / k^{\times}$. By the uniqueness statement in the fundamental theorem of projective geometry, there exists a unique $\alpha \in k^{\times}$such that $\Phi \circ m_{x^{\prime}}=m_{\alpha} \circ m_{\Phi\left(x^{\prime}\right)} \circ \Phi$. The normalisation $\Phi(1)=1$ forces $\alpha=1$, so the two maps are equal.

Proposition 6.5. In the situation of Theorem B, given $\lambda: G_{F \mid k} \stackrel{\sim}{\longrightarrow} G_{F^{\prime} \mid k^{\prime}}$, let $K \mid k$ and $K^{\prime} \mid k^{\prime}$ be corresponding function fields. Then $\lambda$ induces an isomorphism

$$
\lambda^{*}: K^{\prime \times} / k^{\prime \times} \stackrel{\sim}{\longrightarrow} K^{\times} / k^{\times}
$$

of abelian groups which is simultaneously a collineation of projective spaces. If $K_{1} \subseteq K_{2}$ and $K_{1}^{\prime} \subseteq K_{2}^{\prime}$ have the same inseparable degree, the following square commutes:

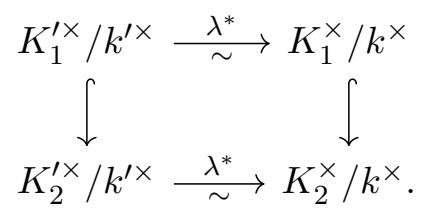

Proof. A divisor $D \in \operatorname{Div}(C)$ is principal if and only if there exist a morphism $\phi: C \rightarrow \mathbb{P}^{1}$ and two points $Q_{0}, Q_{1} \in \mathbb{P}^{1}(k)$ such that $D=\phi^{*}\left(Q_{0}\right)-\phi^{*}\left(Q_{1}\right)$, thus by Lemma 6.1 the isomorphism 
$\lambda^{*}: \operatorname{Div}\left(C^{\prime}\right) \cong \operatorname{Div}(C)$ restricts to the subgroups of principal divisors and induces $\lambda^{*}$ as claimed. The lines in $\operatorname{PDiv}(C)$ are given by

$$
D+\left\{\phi^{*}(Q)-\phi^{*}\left(Q_{0}\right) \mid Q \in \mathbb{P}^{1}(k)\right\}
$$

for a principal divisor $D \in \operatorname{PDiv}(C)$, a morphism $\phi: C \rightarrow \mathbb{P}^{1}$ and a point $Q_{0} \in \mathbb{P}^{1}(k)$. Indeed, a line in $\operatorname{PDiv}(C)$ corresponds to a 2-dimensional $k$-subspace $\langle f, g\rangle \subseteq K$, and if $\phi: C \rightarrow \mathbb{P}^{1}$ is the morphism given by $f / g$, the line is

$$
\begin{aligned}
\left\{\operatorname{div}(a f+b g) \mid(a: b) \in \mathbb{P}^{1}\right\} & =\operatorname{div}(g)+\left\{\operatorname{div}(a f / g+b) \mid(a: b) \in \mathbb{P}^{1}\right\} \\
& =\operatorname{div}(g)+\left\{\phi^{*}(-b: a)-\phi^{*}(\infty) \mid(a: b) \in \mathbb{P}^{1}\right\} .
\end{aligned}
$$

Thus, again by Lemma $6.1, \lambda^{*}$ is a collineation. The commutativity of the square follows from the same lemma.

Hence in the situation of Theorem B, given $\lambda: G_{F \mid k} \stackrel{\sim}{\longrightarrow} G_{F^{\prime} \mid k^{\prime}}$, we have for every pair of corresponding function fields $K \mid k$ and $K^{\prime} \mid k^{\prime}$ an isomorphism

$$
\sigma_{K, K^{\prime}}: K^{\prime}\left|k^{\prime} \stackrel{\sim}{\longrightarrow} K\right| k .
$$

Lemma 6.6. Given $\lambda: G_{F \mid k} \stackrel{\sim}{\longrightarrow} G_{F^{\prime} \mid k^{\prime}}$, there exists a map $K \mapsto K^{\prime}$ from the set of function fields in $F$ to the set of function fields in $F^{\prime}$ such that $\lambda^{-1}\left(U_{K^{\prime}}\right)=U_{K}$ for all $K$, and whenever $K_{1} \subseteq K_{2}$, then $K_{1}^{\prime} \subseteq K_{2}^{\prime}$ of the same inseparable degree.

Proof. Assume char $(k)=p>0$. Fix a function field $K_{0}$ in $F$ and choose any corresponding $K_{0}^{\prime}$. For every $K \supseteq K_{0}$, choose $K^{\prime}$ in its perfect equivalence class such that $\left[K^{\prime}: K_{0}^{\prime}\right]_{i}=\left[K: K_{0}\right]_{i}$. Then for an arbitrary function field $K$ in $F$, choose $K^{\prime}$ in its perfect equivalence class such that $\left[\left(K K_{0}\right)^{\prime}: K^{\prime}\right]_{i}=\left[K K_{0}: K\right]_{i}$. One verifies the assertions by looking at the field diagram

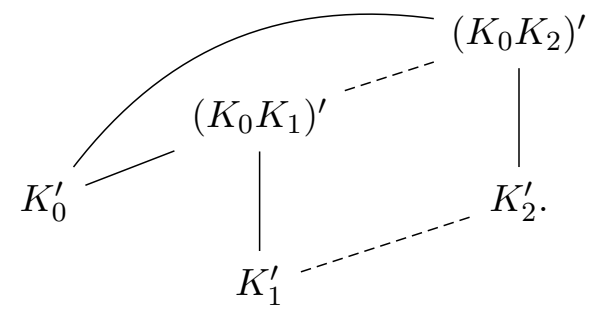

The fields are chosen for the solid lines to have inseparable degrees matching those of the corresponding extensions in $F$. It follows that the same holds for the dashed lines.

Choosing a map $K \mapsto K^{\prime}$ according to the lemma, the isomorphisms $\sigma_{K, K^{\prime}}$ from (6.1) are compatible with each other, hence define $\sigma: F^{\prime}\left|k^{\prime} \stackrel{\sim}{\longrightarrow} F\right| k$ that satisfies $\sigma\left(K^{\prime}\right)=K$ for all function fields $K$ in $F$. It remains to show that the induced isomorphism $\Phi(\sigma): G_{F \mid k} \stackrel{\sim}{\longrightarrow} G_{F^{\prime} \mid k^{\prime}}$ coincides with the given $\lambda$.

Lemma 6.7. Let $F \mid k$ be an extension of algebraically closed fields with $\operatorname{trdeg}(F \mid k)=1$.

(a) Suppose $\sigma \in \operatorname{Aut}(F)$ satisfies $\sigma k=k$ and $\sigma K=K$ for all function fields $K$ in $F$. Then $\sigma=\mathrm{id}$

(b) Assume $\operatorname{char}(k)=p>0$ and suppose $\sigma \in \operatorname{Aut}(F)$ satisfies $\sigma k=k$ and $\sigma K^{i}=K^{i}$ for all function fields $K \mid k$ in $F$. Then $\sigma$ is an integral power of the Frobenius automorphism.

(c) Let $\lambda \in \operatorname{Aut}\left(G_{F \mid k}\right)$ be a topological automorphism such that $\lambda(U)=U$ for all compact open subgroups $U$ in $G_{F \mid k}$. Then $\lambda=\mathrm{id}$.

Proof. (a) Let $x \in F \backslash k$, use it as a coordinate on $\mathbb{P}^{1}$. For every function field extension $k(x) \subseteq K$, corresponding to a morphism $\phi: C \rightarrow \mathbb{P}^{1}$, the automorphism $\sigma$ permutes the normalised discrete valuations of $k(x) \mid k$ that ramify in $K$, i.e. the branch points of $\phi$. But every two-element subset of $\mathbb{P}^{1}(k)$ is the branch locus of some $\phi$, so $\sigma$ must 
act trivially on the set of normalised discrete valuations of $k(x) \mid k$. Therefore we have $\sigma y / y \in k^{\times}$for all $y \in k(x)^{\times}$and we conclude $\sigma=$ id on $k(x)$ by Lemma 2.3. Since $x$ was arbitrary, $\sigma=$ id on $F$.

(b) For each function field $K$, there exists a unique $n \in \mathbb{Z}$ such that $\sigma K=K^{p^{n}}$. We claim that $n$ is independent of $K$. Indeed, if one function field is contained in another, $K_{1} \subseteq K_{2}$, we find $n_{1}=n_{2}$ by looking at generalised inseparable degrees of $K_{1} \subseteq K_{2}$ and $\sigma K_{1} \subseteq \sigma K_{2}$. The general case follows since any two function fields are contained in a common finite extension. Now $\left(\sigma \circ \mathrm{Frob}^{-n}\right)(K)=K$ for all function fields $K$, thus $\sigma=$ Frob $^{n}$ by (a).

(c) Let $\sigma \in G_{F \mid k}$. For all compact open subgroups $U$ of $G_{F \mid k}$ we have

$$
\sigma U \sigma^{-1}=\lambda\left(\sigma U \sigma^{-1}\right)=\lambda(\sigma) U \lambda(\sigma)^{-1},
$$

thus $\sigma^{-1} \lambda(\sigma)$ is contained in the normaliser of $U$. This implies $\sigma^{-1} \lambda(\sigma)\left(K^{i}\right)=K^{i}$ for all function fields $K \mid k$ in $F$, so $\sigma^{-1} \lambda(\sigma)$ is the identity in characteristic 0 and a power of the Frobenius in positive characteristic by (a) and (b), respectively. But it fixes $k$ elementwise, hence $\sigma^{-1} \lambda(\sigma)=1$.

For the isomorphism $\sigma: F^{\prime}\left|k^{\prime} \stackrel{\sim}{\longrightarrow} F\right| k$ constructed from $\lambda: G_{F \mid k} \stackrel{\sim}{\longrightarrow} G_{F^{\prime} \mid k^{\prime}}$, we have

$$
\Phi(\sigma)\left(U_{K}\right)=\sigma^{-1} U_{K} \sigma=U_{\sigma^{-1} K}=U_{K^{\prime}}=\lambda\left(U_{K}\right)
$$

for all function fields $K$ in $F$. Thus $\Phi(\sigma)^{-1} \circ \lambda$ satisfies the hypotheses of Lemma 6.7 (c) and we conclude $\lambda=\Phi(\sigma)$, finishing the proof of Theorem B.

\section{REFERENCES}

[Art57] Emil Artin, Geometric algebra, Interscience Publishers, New York, 1957.

[Bog91] Fedor A. Bogomolov, On two conjectures in birational algebraic geometry, ICM-90 Satellite Conference Proceedings, Springer, Japan, 1991, pp. 26-52 (English).

[BT08] Fedor Bogomolov and Yuri Tschinkel, Reconstruction of function fields, Geom. Funct. Anal. 18 (2008), no. 2, 400-462. MR 2421544

[EP05] Antonio J. Engler and Alexander Prestel, Valued fields, Berlin: Springer, 2005 (English).

[Har77] Robin Hartshorne, Algebraic geometry, Springer, Berlin Heidelberg New York, 1977.

[Har95] David Harbater, Fundamental groups and embedding problems in characteristic $p$, Recent developments in the inverse Galois problem, Contemp. Math., vol. 186, American Mathematical Society, Providence, RI, 1995, pp. 353-369.

[Jac64] Nathan Jacobson, Lectures in abstract algebra. Vol III: Theory of fields and Galois theory, D. Van Nostrand Co., Inc., Princeton, N.J.-Toronto, Ont.-London-New York, 1964. MR 0172871

[Pop90] Florian Pop, On the Galois theory of function fields of one variable over number fields, J. Reine Angew. Math. 406 (1990), 200-218. MR 1048241

[Pop95] Étale Galois covers of affine smooth curves. The geometric case of a conjecture of Shafarevich. On Abhyankar's conjecture, Inventiones Mathematicae 120 (1995), no. 3, 555-578.

[Pop12] _ Recovering function fields from their decomposition graphs, Number theory, analysis and geometry, Springer, New York, 2012, pp. 519-594. MR 2867932

[PŠŠ66] I. I. Pjateckiı̌-Šapiro and I. R. Šafarevič, Galois theory of transcendental extensions and uniformization, Izv. Akad. Nauk SSSR Ser. Mat. 30 (1966), 671-704, In Russian. With an English Translation in Amer. Soc. Math. Translations, 69:111-145, 1968. MR 0202721 (34 \#2581)

[Rov03] M. Rovinsky, On certain isomorphisms between absolute Galois groups, Compositio Mathematica 136 (2003), no. 1, 61-67. MR 1965740 (2004b:12007)

[Rov05] _ Motives and admissible representations of automorphism groups of fields, Math. Z. 249 (2005), no. 1, 163-221. MR 2106976

[Ser97] Jean-Pierre Serre, Galois cohomology, Springer-Verlag, Berlin, 1997, Translated from the French by Patrick Ion and revised by the author. MR 1466966

[Shi71] G. Shimura, Introduction to the arithmetic theory of automorphic functions, Kanô memorial lectures, Princeton University Press, 1971.

Institut für Mathematik, Johann Wolfgang Goethe-Universität, Robert-Mayer-Str. 6-8, 60325 Frankfurt am Main, Germany

E-mail address: luedtke@math.uni-frankfurt.de 